\title{
ISAR IMAGE SEQUENCE BASED AUTOMATIC TARGET RECOGNITION BY USING A MULTI-FRAME MARKED POINT PROCESS MODEL
}

\author{
Csaba Benedek
}

\author{
Computer and Automation Research Institute \\ Distributed Events Analysis Research Group \\ H-1111, Budapest, Kende utca 13-17, Hungary
}

\author{
Marco Martorella \\ University of Pisa \\ Department of Information Engineering \\ Via Caruso 16, I-56122 Pisa, Italy
}

\begin{abstract}
In this paper we propose a Multi-frame Marked Point Process model for automatic target detection and tracking in Inverse Synthetic Aperture Radar (ISAR) image sequences. For purposes of dealing with high ISAR noise, we obtain the optimal target sequence by an energy minimization process, which simultaneously considers the observed image data and prior geometric interaction constraints between the target appearances in the consecutive frames. Finally, a robust permanent scatterer detetection step is introduced to support the target identification process. Evaluation is performed on real ISAR image sequences of ship targets.
\end{abstract}

\section{INTRODUCTION}

Automatic detection, tracking and characterization of ship scattering centers in airborne Inverse Synthetic Aperture Radar (ISAR) image sequences are key tasks of Automatic Target Recognition (ATR) systems that make use of ISAR data. ISAR images are often used for classifying and recognizing targets, since they can provide useful two-dimensional features, where other imaging techniques, such as SAR processing, fail [1,2]. A number of ATR techniques based on sequences of ISAR images have been proposed in the literature. Some of them directly utilize the 2D ISAR images [3], whereas others attempt a 3D signal reconstruction before dealing with the classification problem $[4,5]$. Due to the physics of ISAR imaging, consecutive images of an ISAR sequence may have significantly different quality in terms of image focus. This problem usually leads to a frame selection to discard some bad quality frames [3], which could occur in large numbers in real ISAR image sequences. On the other hand, 3D model reconstruction from ISAR images is still not a very robust technique, which can easily lead to distorted 3D structures and presence of artifacts (false scatterers). For this reason, we propose a robust multi-frame technique, which

This work was supported by the APIS Project of the EDA. The work of the first author was also funded by the János Bolyai Research Fellowship of the Hungarian Academy of Sciences integrates the noisy image information with prior constraints of target shape persistency and smooth motion.

Recently, Marked Point Processes (MPP) [6, 7] have become popular in object recognition tasks, since they can efficiently model the noisy spectral appearance and the geometry of a target using a joint configuration energy function. However, conventional MPP models deal with the extraction of static objects in single images [6] or a pair of remotely sensed photos [7]. Conversely, in the addressed scenario, a moving target must be followed across several frames. Thus, we construct a novel Multiframe MPP ( $\left.\mathrm{F}^{m} \mathrm{MPP}\right)$ framework which simultaneously considers data-object consistency in the individual ISAR images and interactions between objects in the consecutive frames.

Besides the target scatterer's extraction, another issue is to detect characteristic features in the ship objects which provide relevant information for the identification process. For this purpose, we identify permanent bright points in the imaged targets, which are produced by stronger scatterer responses from the illuminated ship. Due to the presence of speckle, image defocus and scatterer scintillation, a significant number of missing and false scatters appear in the individual frames. Permanent scatters are identified by applying a kernel density estimation for the empirical distance histograms.

The outline of the paper is as follows. In Sec. 2 we introduce the proposed $\mathrm{F}^{m}$ MPP model for ship scatterer's detection based on multiple ISAR frames. In Sec. 3, we briefly summarize the permanent scatter extraction and tracking algorithm. Results of ships sequence extraction and ship classification are provided in Sec. 4, while concluding remarks are given in Sec. 5.

\section{MULTIFRAME MARKED POINT PROCESS MODEL}

The input of the proposed algorithm is a sequence of 2D ISAR images, which contains a single ship target. Ship side views in ISAR images are often used as inputs of ATR systems, as they can provide useful features of the objects, such as length or orientation. However, we usually get only limited infor- 

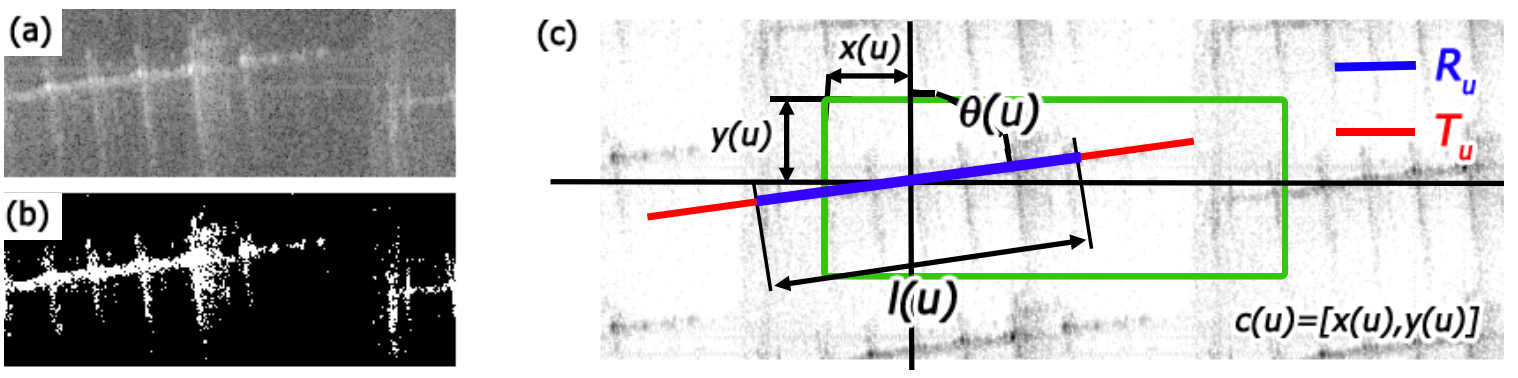

Fig. 1. Target representation in an ISAR image: (a) input image with a single ship object (b) binarized image (c) duplicated image and target fitting parameters. Original image border is shown by the green rectangle

mation about the ship's superstructure. For this reason, we model the imaged targets by line segments in the proposed approach. A frame of a considered input sequence is shown as a grayscale image in Fig. 1(a), after a logarithmic mapping of the raw ISAR data followed by linear scaling. We use in the following binarized inputs (shown in Fig. 1(b)) obtained by thresholding. We must also consider that the ISAR image is periodic both in the horizontal and vertical directions, thus, if the line segment is not center-aligned it may 'break' into two (or four) pieces, as shown in Fig. 1. Therefore, we search for the optimal connected line segment in a duplicated mosaic image, shown in Fig. 1(c), where the central Rectangle of Interest (ROI) corresponds to the original input frame.

Let us denote by $S$ the pixel lattice of the images of the $n$-frame-long ISAR image sequence, and by $s \in S$ a single pixel. We denote by $u_{t}$ a target candidate in frame $t \in$ $\{1,2, \ldots, n\}$. Each target $u$ is described by the $c(u)$ center pixel, $l(u)$ length and $\theta(u)$ orientation parameters (see Fig. 1(c)). Let us denote by $H$ the object space. $B_{t}(s) \in\{0,1\}$ marks the binarized ISAR input value of pixel $s$ in frame $t$. The goal is to obtain a $\omega=\left\{u_{1}, u_{2}, \ldots, u_{n}\right\} \in H^{n}$ target sequence, which we call configuration in the following. We introduce a non-stationary data-dependent $\Phi_{D}(\omega)$ energy function on the configuration space:

$$
\Phi_{D}(\omega)=\sum_{t=1}^{n} A_{D}\left(u_{t}\right)+\gamma \cdot \sum_{t=1}^{n-1} I\left(u_{t}, u_{t+1}\right)
$$

where $A_{D}\left(u_{t}\right)$ is the data-dependent unary object potential, and $I\left(u_{t}, u_{t+1}\right)$ is the prior interaction potential function between objects of consecutive frames. $\gamma$ is a positive weighting factor between the two terms.

We aim to find the Maximum Likelihood (ML) configuration estimate $\widehat{\omega}$, which is obtained as $\widehat{\omega}=\operatorname{argmin}_{\omega \in H^{n}} \Phi_{D}(\omega)$. The $A_{D}\left(u_{t}\right)$ unary potential characterizes a proposed object candidate in the $t$-th frame depending on the local ISAR image data, but independently of other frames of the sequence. Let us first denote by $L_{u} \subset S$ the set of pixels lying under the line of $u$ in the duplicated image. Let us denote by $R_{u} \subset L_{u}$ the pixels covered by the line segment $u$ (see Fig.

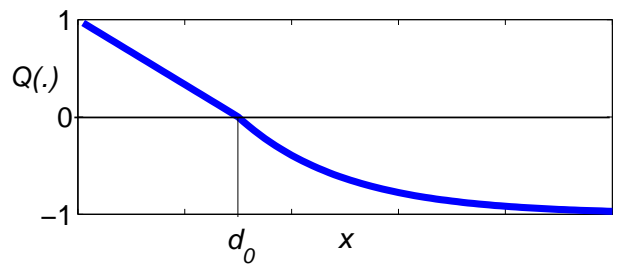

Fig. 2. Plot of the $Q\left(x, d_{0}\right)$ function

1(c)): $R_{u}=\left\{s=\in L_{u} \mid d(s,[x(u), y(u)])<l(u) / 2\right\}$ and by $T_{u} \subset L_{u} \backslash R_{u}$ the pixels of the $L_{u}$ which lie outside the $u$ segment but close enough to its endpoints. Then the unary potential of $u$ is obtained as:

$A_{D}\left(u_{t}\right)=$

$Q\left(\frac{1}{\operatorname{Ar}\left\{R_{u_{t}} \cup T_{u_{t}}\right\}} \sum_{s \in R_{u_{t}}} B_{t}(s)+\sum_{s \in T_{u_{t}}}\left(1-B_{t}(s)\right), d_{0}\right)$,

where a non-linear monotonously decreasing $Q\left(x, d_{0}\right)$ function [7] is used to map the feature domain to the $[-1,1]$ interval (see Fig. 2):

$$
\mathcal{Q}\left(x, d_{0}\right)= \begin{cases}\left(1-\frac{x}{d_{0}}\right), & \text { if } x<d_{0} \\ \exp \left(-10 \cdot\left(x-d_{0}\right)\right)-1, & \text { if } x \geq d_{0}\end{cases}
$$

On the other hand, we assume a persistent frame rate, where the displacement of objects is small between two consecutive frames. Since, due to the imaging technique, the $c(u)$ center is not relevant regarding the real target position, we only penalize large differences of the $\theta(u)$ angle and $l(u)$ length parameters between neighboring objects of the sequence. Thus, the prior interaction term is calculated as:

$I\left(u_{t}, u_{t+1}\right)=\delta_{\theta} \cdot\left|\theta\left(u_{t}\right)-\theta\left(u_{t+1}\right)\right|+\delta_{l} \cdot\left|l\left(u_{t}\right)-l\left(u_{t+1}\right)\right|$

where $\delta_{\theta}>0$ and $\delta_{l}>0$ are weighting parameters. 

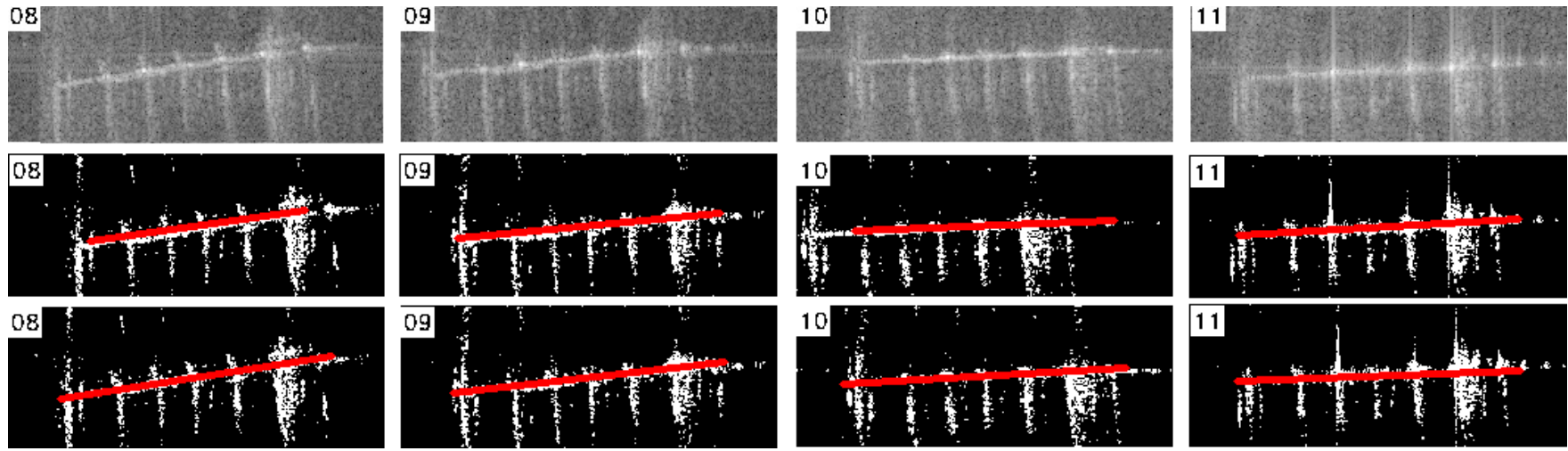

Fig. 3. Center alignment and target line extraction results on Frames 08-11 of a thirteen-frame-long ISAR image sequence. Top: input sequence. Center: frame-by-frame detection. Bottom: detection by the proposed $\mathrm{F}^{m}$ MPP model.

\section{OPTIMIZATION}

We estimate the optimal configuration $\widehat{\omega}$ in a two-step process, which consists of an initialization step and an iterative stochastic refinement step, which is a modification of [6].

The initial step processes the frames of the sequence independently. We extract in each frame's duplicated mosaic image (Fig. 1(c)) the dominant line by applying Hough transform [8], and we consider the longest connecting segment of this line as the initial object candidate. However, as examples in the second row of Fig. 3 show, due to weak foregroundbackground separation, some of the ship segments may be broken and false foreground blobs can also corrupt this direct detection process. For this reason, in a second phase of the detection algorithm we apply and iterative refinement process. We visit the target appearances $u_{t}$ in the consecutive frames one after another, and for each $t$ we propose an alternative $u_{t}^{*}$ candidate by randomly perturbating $u_{t}, u_{t-1}$ or $u_{t+1}$. Then, we calculate the $\Delta \Phi_{D}\left(\omega, u_{t}, u_{t}^{*}\right)$ energy difference between the original configuration $\omega$ and the proposed one $\omega^{*}$ where we replaced $u_{t}$ by $u_{t}^{*}$. We calculate a $d_{\omega}\left(u_{t}, u_{t}^{*}\right)$ exchange probability value according to a monotonously decreasing function of $\Delta \Phi_{D}\left(\omega, u_{t}, u_{t}^{*}\right)$. Finally, we exchange $u_{t}$ to $u_{t}^{*}$ with a probability $d_{\omega}\left(u_{t}, u_{t}^{*}\right)$, continue the above process iteratively till convergence is obtained in the global configuration.

\section{DOMINANT SCATTERER DETECTION AND TRACKING}

In this section, we aim to extract stable characteristic feature points from the ISAR image sequence, which correspond to responses from the target's dominant scatterers. In general, dominant scatterers cause salient bright blobs in the ISAR images; however, as Fig. 5(b) confirms, we should handle numerous missing and false alarms. To overcome the problem, we can exploit the geometrical persistence of the target. Since

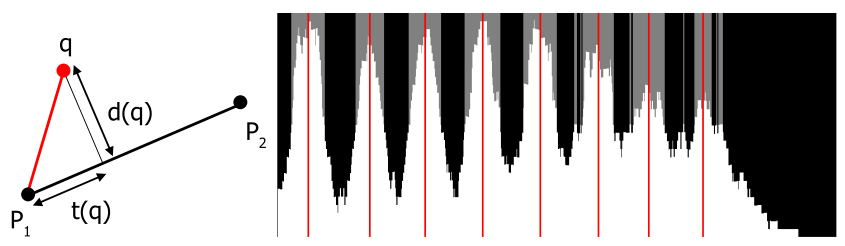

(a)

(b)

Fig. 4. Dominant scatter detection: (a) model parametrization (b) $t(q)$ distance histogram over the ISAR sequence of Fig. 5

dominant scatters correspond to static parts of the ship, we can expect stable relative scatter positions within the object line segment. In the proposed $\mathrm{F}^{m} \mathrm{MPP}$ model, we consider a scatter $q$ as a child-object of a target $u$, which is described by the relative line directional position, $t(q)$, and the signed distance, $d(q)$ from the center line of the parent object $u$, as shown in Fig. 4(a). Dominant scatter positions can be estimated by peak detection in the global $t(q)$ histogram (Fig. 4(b)), calculated for all bright scatter candidates (Fig. 5(b)) of the sequence. Gaussian kernel functions have been applied to filter the significantly noisy input data in the histogram calculation process. Finally, the extracted dominant scatters can be projected back to the coordinate systems of the individual frames (Fig. 5(d)).

\section{EXPERIMENTAL RESULTS}

We have tested our method on four airborne ISAR image sequences containing different ship targets. Object line segment extraction results in four consecutive frames of a selected sequence are shown in Fig. 3: we can observe that the proposed multiframe optimization process can notably improve the initial frame-by-frame detection. For quantitative evaluation, we have manually prepared Ground Truth data and matched the 

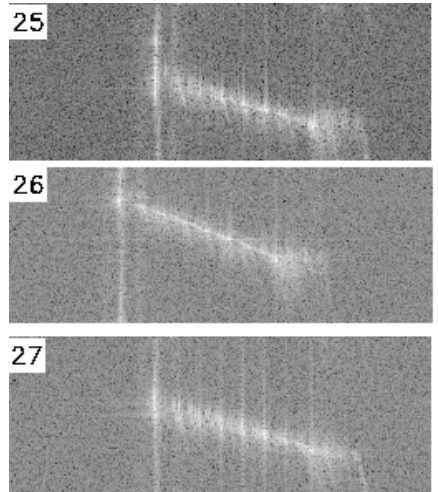

(a)
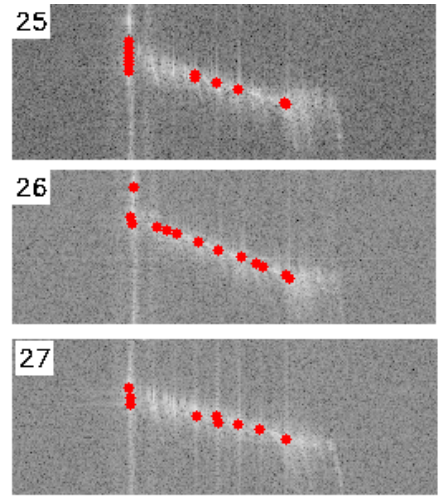

(b)

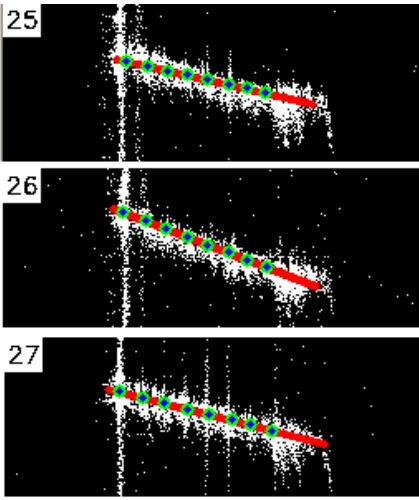

(c)
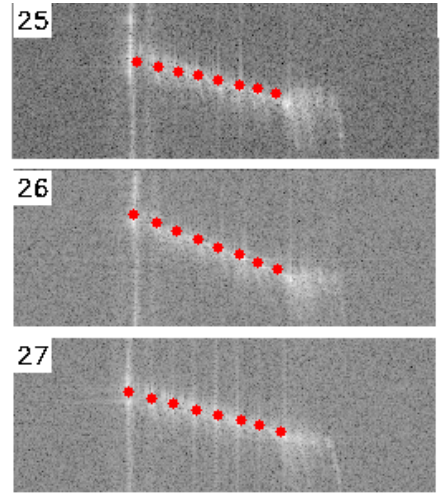

(d)

Fig. 5. Dominant scatter detection (a) ISAR images; (b) initial intensity based detection; (c)-(d) detection after multiframe optimization: (c) scatters projected to the extracted object line (d) scatters projected to the original input

Table 1. $E\left(\left\{u_{t}\right\},\left\{u_{t}^{\mathrm{gt}}\right\}\right)$ error rates of the initial detection (Init Err.) and optimized detection steps ( $\mathrm{F}^{m} \mathrm{MPP}$ Err.)

\begin{tabular}{|l|c|c|c|}
\hline Sequence & Frames & Init Err. & $\mathrm{F}^{m}$ MPP Err. \\
\hline \hline Ship 1 & 13 & 52.0 & 7.5 \\
\hline Ship 2 & 13 & 67.1 & 37.8 \\
\hline Ship 3 & 13 & 17.2 & 12.8 \\
\hline Ship 4 & 54 & 43.7 & 12.6 \\
\hline
\end{tabular}

detection results to the GT. The error measure is defined as:

$$
\begin{aligned}
E\left(\left\{u_{t}\right\},\left\{u_{t}^{\mathrm{gt}}\right\}\right)= & \sum_{t=1}^{n}\left(\left|x\left(u_{t}\right)-x\left(u_{t}^{\mathrm{gt}}\right)\right|+\left|y\left(u_{t}\right)-y\left(u_{t}^{\mathrm{gt}}\right)\right|+\right. \\
& \left.+\left|l\left(u_{t}\right)-l\left(u_{t}^{\mathrm{gt}}\right)\right|+\left|\theta\left(u_{t}\right)-\theta\left(u_{t}^{\mathrm{gt}}\right)\right|\right)
\end{aligned}
$$

where $x, y$ and $l$ are measured in pixels and $\theta$ in degrees. Results in Table 1 confirm that the proposed $\mathrm{F}^{m}$ MPP optimization process reduces the errors significantly.

We demonstrate the dominant scatterer tracking procedure in Fig. 5. Although intensity based feature point detection is very noisy in the individual frames (Fig. 5(b)), the eight permanent scatters are correctly identified by the histogram based technique (see Fig. 4(b) and 5(c)-(d)).

\section{CONCLUSION}

This paper has addressed the detection and classification of ship targets in ISAR image sequences using an energy minimization approach. We have shown that the proposed Multi-frame Marked Point Process schema outperforms the frame-by-frame direct detection techniques, while a permanent scatterer detection algorithm based on histograming technique may efficiently contributes to target classification.

\section{REFERENCES}

[1] J. L. Walker, "Range-doppler imaging of rotating objects," IEEE Trans. Aerospace and Electronic Systems, vol. 16, pp. 23-52, 1980.

[2] D. A. Ausherman, A. Kozma, J. L. Walker, H. M. Jones, and E. C. Poggio, "Developments in radar imaging," IEEE Trans. Aerospace and Electronic Systems, vol. 20, pp. 363-400, 1984.

[3] A. Maki and K. Fukui, "Ship identification in sequential ISAR imagery," Mach. Vision Appl., vol. 15, pp. 149155, 2004.

[4] T. Cooke, "Ship 3D model estimation from an ISAR image sequence," in Proc. IEEE Int. Radar Conf., 2003, pp. $36-41$.

[5] T. Cooke, M. Martorella, B. Haywood, and D. Gibbins, "Use of 3D ship scatterer models from ISAR image sequences for target recognition," Elsevier DSP, vol. 16, pp. 523-532, 2006.

[6] X. Descombes, R. Minlos, and E. Zhizhina, “Object extraction using a stochastic birth-and-death dynamics in continuum," J. Math. Imaging and Vision, vol. 33, pp. 347-359, 2009.

[7] C. Benedek, "Efficient building change detection in sparsely populated areas using coupled marked point processes," in IEEE Geoscience and Remote Sensing Symposium, Honolulu, Hawai, USA, 2010.

[8] S. Guo, T. Pridmore, Y. Kong, and X. Zhang, "An improved hough transform voting scheme utilizing surround suppression," Pattern Recogn. Lett., vol. 30, pp. 12411252, October 2009. 